\title{
Billroth-II with Braun versus Roux-en-Y reconstruction in totally laparoscopic distal gastrectomy for gastric cancer
}

\author{
Feng Chi, Yuefu Lan, Tienan Bi, Shenkang Zhou \\ Department of Gastrointestinal Surgery, Taizhou Hospital, Zhejiang University, Zhejiang, China
}

Videosurgery Miniinv 2021; 16 (4): 664-668

DOI: https://doi.org/10.5114/wiitm.2021.103965

\begin{abstract}
Introduction: Totally laparoscopic distal gastrectomy (TLDG) has been increasingly adopted for the treatment of gastric cancer. Both Billroth-II with Braun (B-IIB) reconstruction and Roux-en-Y $(R-Y)$ reconstruction are commonly performed in TLDG; however, which of these reconstruction techniques is better remains unclear.

Aim: To compare the efficacy of $B-I I B$ reconstruction and $R-Y$ reconstruction in $T L D G$ for gastric cancer.

Material and methods: A total of 105 gastric cancer patients who underwent TLDG with B-IIB or R-Y reconstruction were reviewed from January 2019 to July 2020. Clinicopathological characteristics and perioperative data of the $B$-IIB and $R-Y$ groups were compared.

Results: Clinicopathological characteristics were not significantly different between the $B-I I B$ and $R-Y$ groups. The average total operative time for the $R-Y$ group $(161.9 \pm 20.7 \mathrm{~min})$ was significantly longer than that for the $B$-IIIB group (141.9 $\pm 16.7 \mathrm{~min})$. The average anastomosis time for the $R-Y$ group $(25.5 \pm 4.1 \mathrm{~min})$ was also significantly longer than that for the $B-I I B$ group $(18.9 \pm 3.3 \mathrm{~min})$. Blood loss volume, number of retrieved lymph nodes, time to first flatus, average length of postoperative hospital stay, inflammatory parameters and postoperative complications did not differ between the two groups.

Conclusions: Both $B-I I B$ reconstruction and $R-Y$ reconstruction are safe and effective in $T L D G$. B-IIB reconstruction is easier and faster to perform than $R-Y$ reconstruction in TLDG.
\end{abstract}

Key words: Roux-en-Y, gastric cancer, Billroth-II, Braun anastomosis, totally laparoscopic distal gastrectomy.

\section{Introduction}

To date, surgery remains the only completely curative treatment for gastric cancer. Laparoscopic-assisted distal gastrectomy (LADG) has been applied extensively in the treatment of distal gastric cancer since it was first reported by Kitano [1], providing several advantages such as a small incision, minimal postoperative pain, and fast recovery [2, 3].

In recent years, with the progress of laparoscopic instruments and techniques [4, 5], totally laparoscopic distal gastrectomy (TLDG) has been increasingly adopted for gastric cancer surgery. Advantages of TLDG over LADG have been reported including less invasiveness, better cosmetic results, and a shorter hospital stay [6].

In TLDG, both lymph node dissection and digestive tract reconstruction are performed under the laparoscope, which has higher requirements for surgical skill and experience than LADG, which needs a small epigastrium auxiliary incision to perform digestive tract reconstruction extracorporeally [7].

Presently, both Billroth-II with Braun (B-IIB) reconstruction and Roux-en-Y (R-Y) reconstruction are commonly used methods to establish gastrointestinal continuity in TLDG. However, it is unclear which 
one of these reconstruction methods is better, as few comparative studies of B-IIB reconstruction and $\mathrm{R}-\mathrm{Y}$ reconstruction in TLDG exist.

\section{Aim}

The aim of the study was to compare the efficacy of B-IIB reconstruction and $\mathrm{R}-\mathrm{Y}$ reconstruction in TLDG for gastric cancer.

\section{Material and methods}

This retrospective study recruited consecutive patients who underwent TLDG for gastric cancer in our hospital from January 2019 to July 2020. The hospital's Ethics Committee approved the study (Number: K20200812). Patients with a history of abdominal surgery, those who had undergone neoadjuvant chemotherapy, those who underwent TLDG with Billroth-I (B-I) reconstruction, and those who underwent TLDG combined with other operations were excluded. The included patients were classified into two groups according to the type of reconstruction as the B-IIB group and the R-Y group.

\section{Variables}

Clinicopathological characteristics and perioperative data were evaluated. Patient clinicopathologic characteristics included age, sex, body mass index (BMI), American Society of Anesthesiologists (ASA) status, tumor location, tumor size, pathological stage $\left(7^{\text {th }}\right.$ American Joint Committee on Cancer) [8], and range of lymph node dissection. Perioperative data included total operative time, anastomosis time, blood loss volume, number of retrieved lymph nodes, time to first flatus, postoperative hospital stay, inflammatory parameters and complications. Postoperative complications were evaluated in accordance with the Clavien-Dindo classification [9]. Only complications of grade II or higher were regarded as events. Inflammatory parameters included white blood cell (WBC) count and C-reactive protein (CRP) level measured preoperatively and at 1 and 4 days postoperatively.

\section{Surgical techniques}

The surgical procedures were standardized. Under general anesthesia, one port below the umbilicus for laparoscopy and another four ports in the upper abdomen were used after pneumoperitoneum was instituted. After lymph node dissection performed according to the Japanese treatment guidelines for gastric carcinoma [10], the duodenum was divided close to the pyloric ring, followed by transection of the stomach. For B-IIB reconstruction, gastrojejunostomy was performed about $25 \mathrm{~cm}$ from the Treitz ligament in an antecolic and isoperistaltic fashion. Braun anastomosis was performed about $10 \mathrm{~cm}$ from the gastrojejunostomy. For R-Y reconstruction, the jejunum was divided about $15 \mathrm{~cm}$ from the Treitz ligament after its mesentery had been divided using ultrasonically activated shears. Gastrojejunostomy was performed in an antecolic and isoperistaltic fashion. Jejunojejunostomy was performed $40 \mathrm{~cm}$ distal to the gastrojejunostomy. All the intracorporeal anastomoses were completed using endoscopic staplers.

\section{Statistical analysis}

Continuous variables were presented as means \pm standard deviations and analyzed by Student's $t$ test. Categorical variables were expressed as proportions and tested by the $\chi^{2}$ test or Fisher's exact test. The $p$-values $<0.05$ were considered statistically significant. Analyses were performed using IBM SPSS Statistics version 22.0 (IBM Corp., Armonk, NY, USA).

\section{Results}

A total of 105 gastric cancer patients were included in the study. There were 54 patients in the B-IIB group and 51 in the R-Y group. There were no significant differences in age, sex, BMI, ASA status, tumor location, tumor size, pathological stage, and range of lymph node dissection between the two groups $(p>0.05)$ (Table I).

The average total operative time for the $R-Y$ group was significantly longer than that for the B-IIB group $(p<0.001)$. The average anastomosis time for the R-Y group was also significantly longer than that for the B-IIB group $(p<0.001)$. Blood loss volume, number of retrieved lymph nodes, time to first flatus, length of postoperative hospital stay, and complications did not differ between the two groups $(p>0.05)$ (Table II).

There were no significant differences between the B-IIB and R-Y groups in WBC count measured preoperatively $\left(6.3 \pm 1.2 \times 10^{9} / \mathrm{l}\right.$ vs. $6.4 \pm 1.5 \times 10^{9} /$, $p=0.629)$ and at 1 and 4 days postoperatively (11.6 $\pm 1.0 \times 10^{9} / \mathrm{l}$ vs. $11.4 \pm 1.0 \times 10^{9} / \mathrm{l}, p=0.403 ; 7.1 \pm 1.0 \times$ 
$10^{9} / /$ vs. $6.8 \pm 1.4 \times 10^{9} / \mathrm{l}, p=0.210$ ) (Figure 1 ). There were also no significant differences between the B-IIB and R-Y groups in CRP level measured preop-

Table I. Clinicopathologic characteristics of patients

\begin{tabular}{|c|c|c|c|}
\hline Parameter & B-IIB $(n=54)$ & $\mathrm{R}-\mathrm{Y}(n=51)$ & $P$-value \\
\hline Age [years] & $65.3 \pm 9.8$ & $67.3 \pm 7.9$ & 0.241 \\
\hline Sex: & & & 0.669 \\
\hline Male & $35(64.8)$ & $31(60.8)$ & \\
\hline Female & $19(35.2)$ & $20(39.2)$ & \\
\hline $\mathrm{BMI}\left[\mathrm{kg} / \mathrm{m}^{2}\right]$ & $21.8 \pm 2.4$ & $22.0 \pm 2.6$ & 0.585 \\
\hline ASA status: & & & 0.893 \\
\hline Score I & $24(44.4)$ & $25(49.0)$ & \\
\hline Score II & $24(44.4)$ & $21(41.2)$ & \\
\hline Score III & $6(11.2)$ & $5(9.8)$ & \\
\hline Tumor location: & & & 0.527 \\
\hline Lower & $35(64.8)$ & $36(70.6)$ & \\
\hline Middle & $19(35.2)$ & $15(29.4)$ & \\
\hline Tumor size $[\mathrm{cm}]$ & $3.5 \pm 1.1$ & $3.7 \pm 1.3$ & 0.405 \\
\hline Pathological stage: & & & 0.915 \\
\hline I & $7(13.0)$ & $8(15.7)$ & \\
\hline II & $19(35.2)$ & $18(35.3)$ & \\
\hline III & $28(51.8)$ & $25(49.0)$ & \\
\hline $\begin{array}{l}\text { Range of lymph } \\
\text { node dissection: }\end{array}$ & & & 0.675 \\
\hline D1+ & $5(9.3)$ & $6(11.8)$ & \\
\hline $\bar{D} 2$ & $49(90.7)$ & $45(88.2)$ & \\
\hline
\end{tabular}

$B-\| B-B i l l r o t h-/ l$ with Braun reconstruction, $R-Y-$ Roux-en- $Y$ reconstruction, $B M I$ - body mass index, ASA - American Society of Anesthesiologists. eratively $(2.0 \pm 0.6 \mathrm{mg} / \mathrm{l}$ vs. $1.9 \pm 0.8 \mathrm{mg} / \mathrm{l}, p=0.273)$ and at 1 and 4 days postoperatively $(38.2 \pm 8.6 \mathrm{mg} / \mathrm{l}$ vs. $38.8 \pm 10.3 \mathrm{mg} / \mathrm{l}, p=0.759 ; 58.6 \pm 11.3 \mathrm{mg} / \mathrm{l}$ vs. $62.5 \pm 11.4 \mathrm{mg} / \mathrm{l}, p=0.068$ ) (Figure 2).

\section{Discussion}

The first surgical procedure in TLDG is to resect the distal stomach and perigastric lymph nodes radically, which accounts for primary intraoperative bleeding and embodies the oncological safety of the surgery [11]. In the present study, this procedure in the B-IIB and R-Y groups is almost identical and performed in a standardized way, which could explain the results that the number of retrieved lymph nodes and intraoperative bleeding did not differ between the study groups.

The second surgical procedure in TLDG is digestive tract reconstruction, which still remains a difficult and controversial issue [12, 13]. The ideal digestive tract reconstruction should maintain the normal anatomical and physiological functions of the digestive tract as much as possible to meet functional requirements while ensuring the safety of the anastomosis [14]. Presently, common types of digestive tract reconstruction in TLDG include B-I, B-II, and $R-Y$ reconstructions. The gastrointestinal tract after B-I reconstruction appears anatomically and physiologically closer to the normal compared to the other two types of reconstructions, which can reduce the incidence of complications caused by gastrointestinal dysfunction $[15,16]$. However, B-I reconstruction requires retaining a sufficiently long duodenum and

Table II. Perioperative data of patients

\begin{tabular}{|lccc|}
\hline Parameter & B-IIB $(n=54)$ & R-Y $(n=51)$ & $P$-value \\
\hline Total operative time [min] & $141.9 \pm 16.7$ & $161.9 \pm 20.7$ & $<0.001$ \\
\hline Anastomosis time [min] & $18.9 \pm 3.3$ & $25.5 \pm 4.1$ & $<0.001$ \\
\hline Blood loss volume [ml] & $71.9 \pm 23.8$ & $70.2 \pm 22.2$ & 0.714 \\
\hline Number of retrieved lymph nodes & $33.4 \pm 5.7$ & $32.7 \pm 7.0$ & 0.553 \\
\hline Time to first flatus [days] & $2.1 \pm 0.7$ & $2.2 \pm 0.8$ & 0.744 \\
\hline Postoperative hospital stay [days] & $7.0 \pm 0.6$ & $7.1 \pm 0.7$ & 0.371 \\
\hline Complications: & $3(5.6)$ & $4(7.8)$ & 0.711 \\
\hline Intraabdominal abscess & 1 & 0 & \\
\hline Ileus & 1 & 2 & \\
\hline Pulmonary infection & 0 & 1 & \\
\hline Wound infection & 1 & 1 & \\
\hline
\end{tabular}

B-IIB - Billroth-II with Braun reconstruction, $R-Y-$ Roux-en- $Y$ reconstruction. 


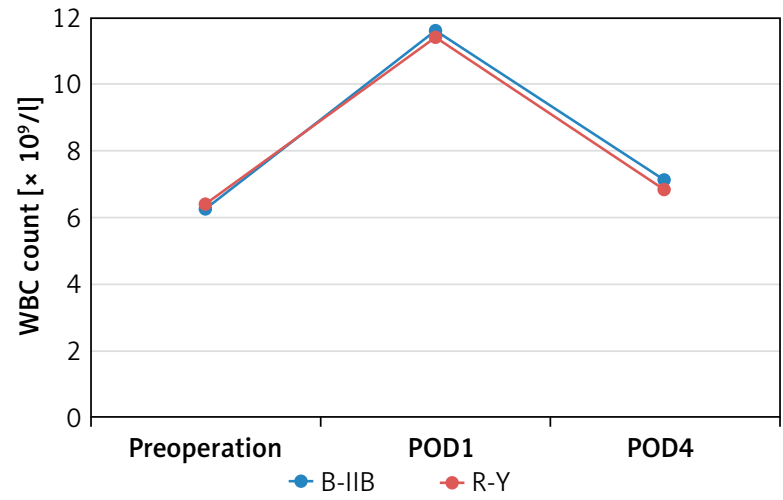

Figure 1. Changes of perioperative white blood cell (WBC) count

B-IIB - Billroth-II with Braun, $R-Y-$ Roux-en-Y, $P O D$ - postoperative day.

more residual stomach to ensure that the anastomotic stoma is unrestricted $[17,18]$. Therefore, this method is highly restricted by the size and location of the tumor. By contrast, B-II and $\mathrm{R}-\mathrm{Y}$ reconstructions have a more adequate scope for the removal of the stomach and thus have a wider range of applications than $\mathrm{B}-\mathrm{I}$ reconstruction.

However, simple B-II reconstruction has a higher incidence of complications than $\mathrm{R}-\mathrm{Y}$ reconstruction such as afferent loop obstruction and intra-abdominal hernia, and the occurrence of alkaline reflux gastritis and anastomotic stomatitis is common [19]. For these reasons, Braun anastomosis can often be performed between the afferent and efferent loops after B-II anastomosis so that bile, duodenal juice, and pancreatic juice can enter directly into the efferent loop [20]. This method could help reduce the incidence of postoperative complications and improve the patients' quality of life. Indeed, in our hospital, we routinely perform Braun anastomosis following B-II anastomosis.

In TLDG, the safety of anastomosis is of primary concern. In our study, no anastomosis-related complications occurred in the study groups. There were no significant differences in postoperative outcomes between the study groups either. These favorable results, which we attribute to the accumulation of operative experience and the use of endoscopic staplers, demonstrate that both B-IIB and $\mathrm{R}-\mathrm{Y}$ reconstructions are safe to perform in TLDG. Intracorporeal anastomosis in TLDG is difficult to perform and requires a high level of experience [21-23]. All surgeons who participated in the current study had abundant prior experience in

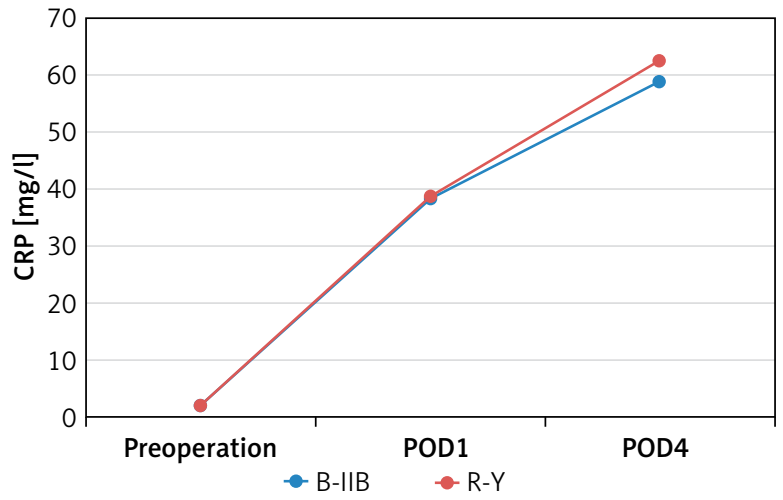

Figure 2. Changes of perioperative C-reactive protein (CRP) level

B-IIB - Billroth-II with Braun, $R-Y-$ Roux-en-Y, $P O D$ - postoperative day.

open gastrectomy and LADG, and had completed the training required for TLDG before beginning the study. All the intracorporeal anastomoses in this study were completed using endoscopic staplers, which can easily enter and exit the abdominal cavity through the trocar and help perform the anastomoses more conveniently than hand-sewing techniques.

In this study, the average total operative time and anastomosis time for the R-Y group were longer than those for the B-IIB group. This is mainly because $\mathrm{R}-\mathrm{Y}$ reconstruction is more complex to perform than $B$-IIB reconstruction, with the additional steps of dividing the jejunum and its mesentery. For this reason, B-IIB reconstruction could be recommended as a better choice for surgeons inexperienced in TLDG.

The major limitation of the current study was its retrospective design. Also, the sample size was not large. Since the study evaluated the short-term outcomes of B-IIB and R-Y reconstructions in TLDG, future prospective studies focusing on the patients' quality of life and long-term survival will be required to further evaluate this issue.

\section{Conclusions}

In this study, the type of reconstruction had no influence on surgical outcomes, which indicates that both $B-I I B$ reconstruction and $R-Y$ reconstruction are safe and effective in TLDG. B-IIB reconstruction is easier and faster to perform than $R-Y$ reconstruction in TLDG, and therefore could be recommended as a priority for surgeons unfamiliar with this technique. 


\section{Acknowledgments}

The present study was supported by the Natural Science Foundation of Zhejiang Province (No. LGF19H160017).

\section{Conflict of interest}

The authors declare no conflict of interest.

\section{References}

1. Kitano S, Iso Y, Moriyama M, et al. Laparoscopy-assisted Billroth I gastrectomy. Surg Laparosc Endosc 1994; 4: 146-8.

2. Takiguchi S, Fujiwara Y, Yamasaki M, et al. Laparoscopy-assisted distal gastrectomy versus open distal gastrectomy. A prospective randomized single-blind study. World J Surg 2013; 37 : 2379-86.

3. Lee JH, Nam BH, Ryu KW, et al. Comparison of outcomes after laparoscopy-assisted and open total gastrectomy for early gastric cancer. Br J Surg 2015; 102: 1500-5.

4. Kirmizi S, Kayaalp C, Karagul S, et al. Comparison of Harmonic scalpel and Ligasure devices in laparoscopic Roux-en-Y gastric bypass. Videosurgery Miniinv 2017; 12: 28-31.

5. Major P, Janik MR, Wysocki M, et al. Comparison of circular- and linear-stapled gastrojejunostomy in laparoscopic Roux-en-Y gastric bypass: a multicenter study. Videosurgery Miniinv 2017 12: $140-6$.

6. Jin HE, Kim MS, Lee CM, et al. Meta-analysis and systematic review on laparoscopic-assisted distal gastrectomy (LADG) and totally laparoscopic distal gastrectomy (TLDG) for gastric cancer: preliminary study for a multicenter prospective KLASSO7 trial. Eur J Surg Oncol 2019; 45: 2231-40.

7. Choi Cl, Lee CM, Park JH, et al. Recent status of laparoscopic distal gastrectomy in korea: a multicenter retrospective cohort study (Pre-study Survey of KLASS-07 Trial). Front Oncol 2019; 9: 982.

8. Washington $\mathrm{K}$. 7th edition of the AJCC cancer staging manual: stomach. Ann Surg Oncol 2010; 17: 3077-9.

9. Dindo D, Demartines N, Clavien PA. Classification of surgical complications: a new proposal with evaluation in a cohort of 6336 patients and results of a survey. Ann Surg 2004; 240: 205-13.

10. Japanese Gastric Cancer Association. Japanese gastric cancer treatment guidelines 2014 (ver. 4). Gastric Cancer 2017; 20: 1-19.

11. Uzun O, Gulmez S, Senger AS, et al. Prognostic factors in operated T3-T4 gastric cancer. J Coll Physicians Surg Pak 2020; 30: 1047-52.

12. Ren Z, Wang WX. Comparison of Billroth I, Billroth II, and RouX en-Y reconstruction after totally laparoscopic distal gastrectomy: a randomized controlled study. Adv Ther 2019; 36: 2997. 3006.

13. Shim JH, Oh SI, Yoo HM, et al. Roux-en-Y gastrojejunostomy after totally laparoscopic distal gastrectomy: comparison with Billorth II reconstruction. Surg Laparosc Endosc Percutan Tech 2014; 24: 448-51.
14. Piessen G, Triboulet JP, Mariette C. Reconstruction after gastrectomy: which technique is best? J Visc Surg 2010; 147: e273-83.

15. Watanabe $Y$, Watanabe $M$, Suehara N, et al. Billroth-I reconstruction using an overlap method in totally laparoscopic distal gastrectomy: propensity score matched cohort study of shortand long-term outcomes compared with Roux-en-Y reconstruction. Surg Endosc 2019; 33: 3990-4002.

16. Toyomasu Y, Ogata K, Suzuki M, et al. Comparison of the physiological effect of Billroth-I and Roux-en-Y reconstruction following laparoscopic distal gastrectomy. Surg Laparosc Endosc Percutan Tech 2018; 28: 328-33.

17. Jeong O, Jung MR, Park YK, et al. Safety and feasibility during the initial learning process of intracorporeal Billroth I (deltashaped) anastomosis for laparoscopic distal gastrectomy. Surg Endosc 2015; 29: 1522-9.

18. Jang CE, Lee SI. Modified intracorporeal gastroduodenostomy in totally laparoscopic distal gastrectomy for gastric cancer: early experience. Ann Surg Treat Res 2015; 89: 306-12.

19. Csendes A, Burgos AM, Smok G, et al. Latest results (12-21 years) of a prospective randomized study comparing Billroth II and Roux-en-Y anastomosis after a partial gastrectomy plus vagotomy in patients with duodenal ulcers. Ann Surg 2009; 249: 189-94.

20. Vogel SB, Drane WE, Woodward ER. Clinical and radionuclide evaluation of bile diversion by Braun enteroenterostomy: prevention and treatment of alkaline reflux gastritis. An alternative to Roux-en-Y diversion. Ann Surg 1994; 219: 458-65.

21. Park K, Lee Y, Kim D. First experience of junior surgeons with laparoscopic distal gastrectomy: in view of comparison with experienced surgeons. Videosurgery Miniinv 2020. doi:10.5114/ wiitm.2020.99310.

22. Ahn CW, Hur H, Han SU, et al. Comparison of intracorporeal reconstruction after laparoscopic distal gastrectomy with extracorporeal reconstruction in the view of learning curve. J Gastric Cancer 2013; 13: 34-43.

23. Kim HG, Park JH, Jeong SH, et al. Totally laparoscopic distal gastrectomy after learning curve completion: comparison with laparoscopy-assisted distal gastrectomy. J Gastric Cancer 2013; 13: 26-33.

Received: 14.12.2020, accepted: 16.01.2021. 